\title{
Salivary Sensors in Point-of-Care Testing
}

\author{
Masaki Yamaguchi* \\ Graduate School of Engineering, Iwate University, \\ 4-3-5 Ueda, Morioka 020-8551, Japan
}

(Received August 26, 2009; accepted March 16, 2010)

Key words: saliva, biosensor, biomarker, point-of-care testing, systemic disease

The key technologies for realizing diagnosis of systemic diseases using saliva samples are biochips, biosensors, and biomarkers. Chemical salivary test (salivary test) has overwhelming advantages over blood test in that it is intrinsically safe and test samples can be self-collected any time. However, a diagnostic methodology for systemic diseases cannot be established on the basis of these advantages alone. It is considered that the practical application of salivary test depends on the successful demonstration that either the diagnostic accuracy of the test is significantly higher than that of conventional techniques, or it can provide additional new information to aid medical treatments. To be specific, this involves the use of new biomarkers. Although rapid, low-cost and highsensitivity analytical techniques using saliva sampling have improved tremendously in recent years, the suggested applications of such techniques are still limited to oral diseases, viral infections and human stress tests. However, in the near future, salivary sensors will be practically applied to various systemic diseases as point-of-care testing (POCT).

\section{Introduction}

Saliva has attracted attention as a human sample that can be collected noninvasively not only for the diagnosis of oral diseases ${ }^{(1)}$ but also for the potential diagnosis of systemic diseases. ${ }^{(2-4)}$ Considerable time has passed since it was first predicted that saliva could become a substitute for blood as a medical test sample (sample). The time may have finally arrived for this prediction to be realized, that is, when saliva can be used in point-of-care testing (POCT). Examples of POCT have become familiar in recent years. For example, the self-monitoring blood glucose meter is now widely used by diabetics and disposable pregnancy testing kits using urine samples have become well established as over-the-counter (OTC) products sold in drug stores. Thus, clinical tests that were previously limited to specialist testing centers could now be replaced by POCT using samples other than blood.

In developing POCT devices, it is necessary to consider eliminating mental and physical pains associated with sampling in addition to the risk of infection from the "Corresponding author: e-mail: masakiy@iwate-u.ac.jp 
samples. In the roadmaps for drug development and diagnosis that are being drawn up in advanced countries, technologies with semi- and non-invasive approaches to sample collection and processing could be realized around 2015 .

The elemental technologies that are required to realize such a diagnostic methodology for systemic diseases using saliva samples are as follows:

(i) Biochip: used for collecting and concentrating saliva samples through a simple and hygienic process to obtain the volume necessary for testing;

(ii) Biosensor: used as a rapid, low-cost, high-sensitivity analytical technology for biomarkers;

(iii) Biomarker: a chemical or substance that can be effectively used for the prevention and diagnosis of diseases, since they are strongly correlated to the severity of the disease. Technical consideration of the influences of ingredients, inhibitors or competitive chemicals is required.

One possibility for the realization of such a diagnostic methodology is based on the development and widespread use of micro-electromechanical systems (MEMS) developed in the semiconductor industry together with the extensive development of bioanalytical techniques employing high-specificity and high-selectivity molecular recognition mechanisms of enzymes, antigens/antibodies, mRNA and DNA.(5) In other words, owing to collaborations between medical and engineering researchers, above criteria (i) and (ii) are no longer constraints.

This review focuses on the current status and future prospects of diagnostic methodologies using saliva samples for detection of systemic diseases. The direction of new applications for salivary sensors is discussed by introducing commercially available salivary tests for oral and systemic diseases.

\section{Methods of Salivary Test}

Table 1 shows the advantages of saliva sampling in POCT for systemic diseases. The principal advantage of salivary test over chemical blood test is its safety. The use of needles in blood collection using cannot be completely free of danger from infection by disease-causing viruses. Since salivary test is a noninvasive technique, then patients who are currently restricted from using needles, such as children, the aged, and hemophiliac

Table 1

Advantages of saliva samples as a diagnostic medium for systemic diseases in POCT.

\begin{tabular}{lccc}
\hline Viewpoint & Saliva & Blood & Urine \\
\hline Safety & High & Low & High \\
Physical pain & N.A. & Significant & N.A. \\
Mental pain & N.A. & Significant & N.A. \\
Danger of infection & N.A. & High & N.A. \\
Self-collection & Easy & Difficult & Moderate \\
Repeated collection & Easy & Difficult & Impossible \\
Economic efficiency & Cheap & Moderate & Cheap \\
\hline
\end{tabular}

*N.A.: not applicable 
patients, will be afforded a number of advantages in self-monitoring. Next, unlike blood, saliva can be self-collected, and unlike urine, saliva can be collected any time. These are the important conditions that POCT must meet in order to make on-site testing possible at home and in the workplace. Furthermore, it is particularly useful for mass screening tests. The influences of ingredients, viscosity and color intensity of each sample on analysis accuracy are different from those of biomarkers.

Table 2 shows approaches to the diagnosis of systemic diseases and body conditions using salivary biomarkers. ${ }^{(6-24)}$ Some salivary biomarkers that could possibly be used for diagnostic methodologies have already been studied and even partly applied to clinical use. They can be classified in terms of origin into antigens/antibodies, hormones, neurotransmitters, genes, enzymes and xenobiotics. Among those listed above, only antibody testing for HIVs (HIV-1 and HIV-2) has been made available commercially (OraSure Technologies, Inc., PA). Development of methods for the proteome (entire complement of proteins expressed by a genome) and transcriptome (set of all messenger RNA (mRNA) molecules) analyses of saliva is currently progressing. Saliva mRNA

Table 2

Approaches to the diagnosis of systemic diseases and body conditions using salivary biomarkers.

\begin{tabular}{|c|c|c|c|}
\hline \multicolumn{2}{|c|}{ Biomarker } & \multirow{2}{*}{ Disease and condition } & \multirow{2}{*}{ Reference } \\
\hline Classification & Chemical & & \\
\hline \multirow[t]{2}{*}{ Antigen and antibody } & HIV & AIDS & ${\text { Ishikawa et al. }{ }^{(6)}}$ \\
\hline & Hepatitis C & Hepatitis & Champion et al..$^{(7)}$ \\
\hline \multirow[t]{5}{*}{ Hormone } & Cortisol & Stress-induced diseases & Vining et al. ${ }^{(8)}$ \\
\hline & Testosterone & & Dabbs et al..$^{(9)}$ \\
\hline & Androstenedione & & Azurmendi et al. ${ }^{(10)}$ \\
\hline & DHEA & & Olff et al..$^{(11)}$ \\
\hline & $\mathrm{E} 2, \mathrm{Pg}$ & & Chatterton et al..$^{(12)}$ \\
\hline Neurotransmitter & $\beta$-Endorphin & Stress-induced diseases & Henning et al..$^{(13)}$ \\
\hline \multirow[t]{4}{*}{ Gene } & mRNA & Cancer & Huang ${ }^{(14)}$ \\
\hline & & & $\mathrm{Hu}$ et al..$^{(15)}$ \\
\hline & & & Zimmermann and Wong ${ }^{(16)}$ \\
\hline & DNA & & Zimmermann et al..$^{(17)}$ \\
\hline \multirow[t]{3}{*}{ Enzyme } & $\alpha$-amylase & Stress-induced diseases & Groza et al. ${ }^{(18)}$ \\
\hline & & & Speirs et al. ${ }^{(19)}$ \\
\hline & & & Yamaguchi et al. ${ }^{(20)}$ \\
\hline \multirow[t]{4}{*}{ Xenobiotics } & Alcohol & Alcoholism & Deguitis et al. ${ }^{(21)}$ \\
\hline & Cotinine & Smoking & Stookey et al. ${ }^{(22)}$ \\
\hline & Phenytoin & Drug abuse & Fu et al..$^{(23)}$ \\
\hline & Cocaine, Codeine & $\begin{array}{l}\text { Prohibited drug, } \\
\text { Narcotic drug }\end{array}$ & Kacinko et al..$^{(24)}$ \\
\hline
\end{tabular}

HIV, human immunodeficiency virus; AIDS, acquired immune deficiency; DHEA, dehydroepiandrosterone; E2, estradiol; Pg, progesterone; mRNA, messenger ribnucleic acid; DNA, deoxyribonucleic acid 
profiles have been obtained using a DNA chip (HG U133A array, Affymetrix, Inc., CA) containing 22,215 human gene cDNA probe sets. ${ }^{(15)}$ Between 2,521 and 3,363 saliva mRNAs have been reliably detected from participants.

For many stress-induced diseases, no objective or quantitative diagnostic standards have yet been established. Some biomarkers that are directly or indirectly related to the sympathetic nervous system and endocrine system can significantly change in concentration depending on the degree of stress and thus be labeled as stress markers. Therefore, stress markers such as cortisol have been studied as a diagnostic index for post-traumatic stress disorder (PTSD), chronic fatigue syndrome (CFS) and irritable bowel syndrome (IBS). In the United States, a business model for the stress testing of commercial pilots is ready for launching. In this business model, the kit is first purchased through the internet. The user then mails in a saliva sample that he/she collected himself/herself and the test results are returned showing the number of stress markers and whether or not the absolute values are within standard range.

Xenobiotics have typically been used for physical condition evaluation rather than for the diagnosis of diseases. Therapeutic drug monitoring (TDM) for antiepileptic drugs and investigations into smoking and drug abuse are typical uses of such applications. A drug-abuse testing system (RapiScan, Cozart Bioscience Ltd., UK) has been used as a method of on-site testing by Immigration Officers at airports in Europe.

Table 3 and Fig. 1 respectively show the principles and schematic diagram of the sensor devices used for the detection of salivary biomarkers. The specificity of these procedures increases in the following order, enzymatic method, antigen-antibody method, hybridization method; however, the cost of the tests increases in the same order. Studies using salivary biomarkers have become very popular following sales of a series of enzyme-linked immunosorbent assay (ELISA) kits for use with saliva samples by Salimetrics, LLC (PA, USA) and other companies. The gold colloid method (Fig. 1(a)) makes a low-cost qualitative test possible by exhibiting the color reactions of ELISA on a visible line on a biochip. Enzyme sensors (Fig. 1(b)), immunosensors and

Table 3

Sensor devices used for the detection of salivary biomarkers.

\begin{tabular}{lll}
\hline Principle of molecular recognition & Salivary biomarker & Sensor device \\
\hline Enzymeatic method & Enzyme, substrate & Test paper (dry chemistry) \\
& & Enzyme sensor (electrochemica sensor) \\
Immunoasay & Antigen and antibody & ELISA \\
& Hormone & Gold colloid method \\
& Neurotransmitter & Electrophoresis \\
& Enzyme & Immuno-sensor (electrochemical sensor) \\
& Xenobiotics & SPR \\
& & LSPR \\
Hybridization & mRNA, DNA & DNA chip \\
\hline
\end{tabular}

ELISA: enzyme-linked immunosorbent assay, LSPR: local surface plasmon resonance, SPR: surface plasmon resonance 
(a)
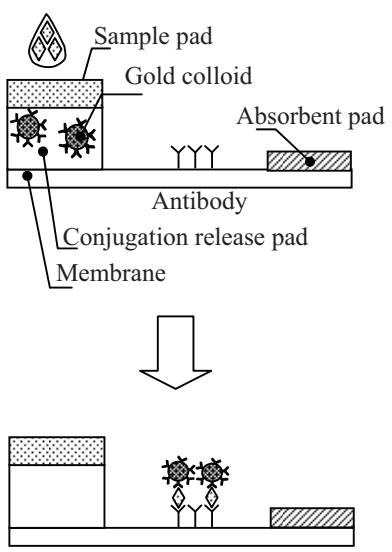

(c)

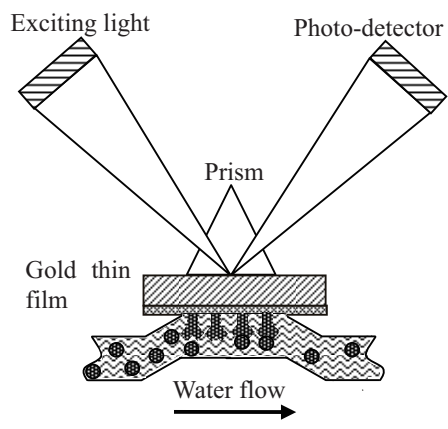

(b)

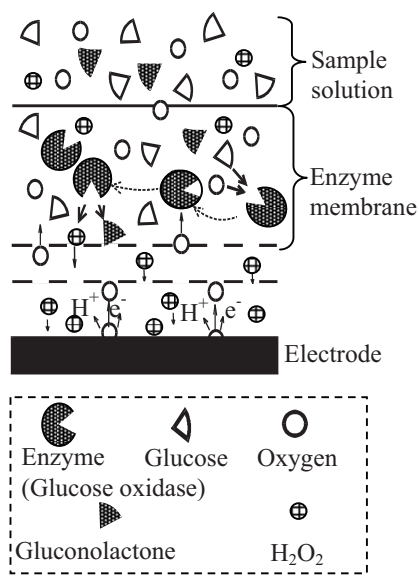

(d)

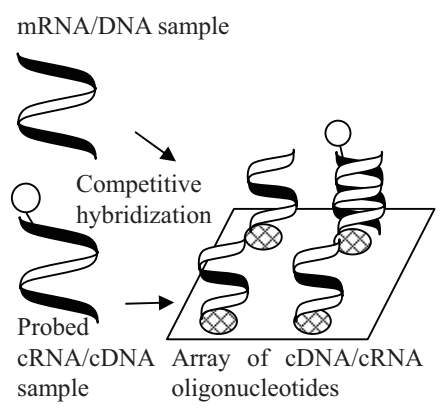

Fig. 1. Schematic diagram of the sensor devices used for the detection of salivary biomarkers: (a) gold colloid method, (b) enzyme sensor, (c) SPR, and (d) DNA chip.

surface plasmon resonance (SPR, Fig. 1(c)) $)^{(25)}$ exhibit the color reactions of the ELISA process by way of electro-chemical or optical phenomena, and thus, high-sensitivity and continuous measurements are possible. A nanoscale optical biosensor based on localized surface plasmon resonance (LSPR) spectroscopy has been developed to monitor the interaction between antigens and antibodies. ${ }^{(26)}$ DNA chips (DNA microarrays) are a new tool used to identify mutations in genes. A DNA chip, which consists of a small glass plate encased in plastic, is manufactured similarly to a computer microchip (Fig. 1(d)). The surface of each chip contains thousands of short, synthetic, single-stranded DNA sequences, which together add up to the normal gene in question when competitive hybridization occurs between the immobilized synthetic cDNA/cRNA strands and mRNA/DNA in a sample. Because chip technology is still relatively new, it is currently only a research tool. 
In order to consider new approaches to diagnostic methodologies using salivary biomarkers, some novel devices that are already on the market are introduced.

\section{Salivary Test of Oral Diseases}

A novel POCT platform (DentoAnalyzer, Dentognostics GmbH, Germany) to quantify micro-organisms that cause dental infections and inflammatory markers reflecting oral disease status has been put on the market in Germany (Fig. 2).(27) This system is based on sandwich immunoassay technology, using a disposable cartridge and is used for the quantitative analysis of salivary markers as measured for the absorbance of light. Thus, the principle is almost the same as that of ELISA. The main component is a small unit that is easily available in dental clinics. Polyhorseradish peroxidaseconjugated antibodies have been developed for detection of dental infections and inflammatory markers, which results in high sensitivity.

The above assay enables a user to quantify 500 colony-forming units of Streptococcus sobrinus (S. sobrinus) per $\mathrm{ml}$ of saliva as a dental infection marker. Streptococcus mutans (S. mutans) $)^{(28)}$ is considered to be the major cause of dental caries. S. mutans and $S$. sobrinus have been widely detected in all ethnic groups. On the other hand, matrix metalloproteinase- 8 (MMP-8) is analyzed as a dental inflammatory marker secreted by periodontium cells. MMP- 8 has been identified as a major tissue-destructive enzyme of the extracellular matrix in periodontal diseases.

The diagnosis of oral diseases using MMP- 8 has been focused on by a relatively large number of researchers. A micro-total analysis system ( $\mu$ TAS) that utilizes MEMS technology to create micro-flow channels and reaction vessels on a biochip to analyze biomarkers from minute volumes of test samples has been fabricated. A prototype $\mu$ TAS for MMP-8 analysis (Sandia Corporation, NM) enables the user to specifically detect

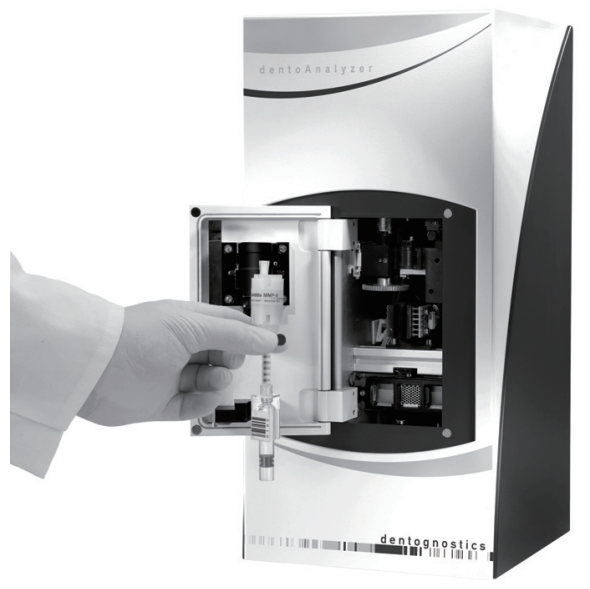

Fig. 2. Immunoassay-based diagnostic technology to quantify micro-organisms causing dental infections and inflammation (DentoAnalyzer, Dentognostics GmbH, Germany). 
MMP-8 with a fluorescence-labeled anti-MMP-8 antibody, and then to separate it by electrophoresis for further fluorescence analysis. This device enables the quantification of MMP-8 within only 10 min from only $20 \mu \mathrm{l}$ of saliva sample. ${ }^{(29)}$ This method exhibits a favorable linearity of $R^{2}=0.979$ compared with conventional ELISA.

\section{Salivary Test of Systemic Diseases}

\subsection{Diagnosis of virus infections}

The DNA and RNA detection of viruses by reverse transcription-polymerase chain reaction (RT-PCR) has already been used for the diagnosis of infections (genetic diagnosis). However, owing to the long time required for the preparation and amplification of DNA/RNA, rapid diagnosis is impossible. Therefore, in an attempt to realize its practical use in POCT, efforts have been made to considerably shorten the time necessary for analysis using immunoassays.

As a diagnostic methodology for systemic diseases, a prototype rapid antigen test for the on-site detection of respiratory syncytial virus (RSV) infection has been developed by OraSure Technologies Inc. (UPlink, PA, Fig. 3) ${ }^{(30)}$ RSV belongs to Paramyxoviridae, the same family as the parainfluenza, measles, and mumps viruses. RSV has been reported worldwide and causes an infection that affects the lungs and causes infant bronchiolitis and pneumonia.

The currently available UPlink was originally developed for the on-site testing of oral samples for drug abuse. UPlink consists of two kinds of disposable devices (UPlink collector and assay cassette) and a monitor with a near-infrared laser (UPlink analyzer). UPlink is based on sandwich immunoassay technology using an RSV monoclonal antibody. Qualitative tests are being conducted to determine whether a test line of a monoclonal antibody can be seen on a cellulose pad placed on the assay cassette. In this way, the need to develop a rapid diagnosis technology for infection arises from the necessity of preventing infectious diseases spreading in today's borderless era.

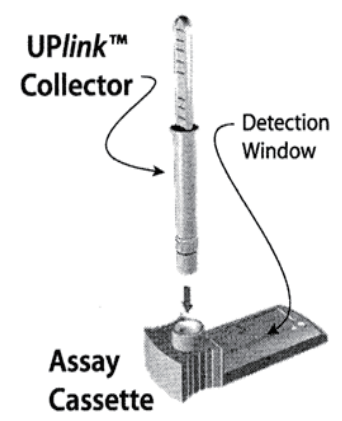

UPlink $^{\text {Tm }}$ Analyzer

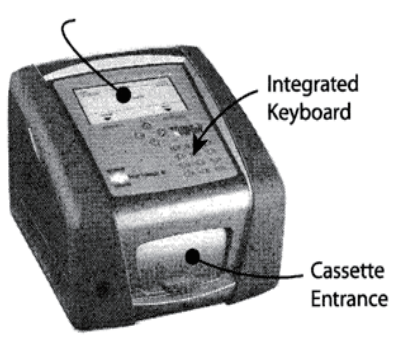

Fig. 3. Prototype of rapid antigen test for the detection of respiratory syncytial virus infection (UPlink, OraSure Technologies, Inc., PA, Ann. N. Y. Acad. Sci. 1098(28)). 


\subsection{Human stress evaluation}

It is widely accepted that psychological stress can produce physiological effects that are similar to those produced by physical challenges. Cortisol is an established biomarker of the hypothalamic-pituitary-adrenocortical (HPA) axis. Salivary cortisol concentration closely correlates to serum cortisol concentration. ${ }^{(31)}$ In contrast, such a well-characterized salivary biomarker related to the activity of the sympathetic nervousadrenalmedullary (SAM) system is still missing. Salivary $\alpha$-amylase activity (SAA) has been found to increase slightly with salivary flow rate, and large increments in amylase concentration have been observed during sympathetic control by Speirs et al. ${ }^{(20)}$ Medication with betablockers significantly suppresses the secretion of SAA, which provides direct evidence of the sensitivity of SAA to changes in adrenergic activation. ${ }^{(32)}$ Currently, it is considered that measurement of SAA is useful for evaluating the SAM system.

To realize a hand-held monitor of the sympathetic nervous system, a completely automated analytical system for measuring SAA using a dry-chemistry system was developed (Amylase monitor, Nipro Co., Japan, Fig. 4). ${ }^{(33)}$ This monitor was made possible by the fabrication of a disposable test strip equipped with built-in collecting and reagent papers and an automatic saliva transfer device. Within an SAA range of 10 $-150 \mathrm{kU} / 1$, the calibration curve for the hand-held monitor showed a coefficient with $R^{2}$ $=0.97$. Accordingly, it was demonstrated that the hand-held monitor enables a user to automatically measure SAA with high accuracy with only $30 \mu 1$ of saliva within a minute from collection to measurement completion.

It is reported that SAA increases more significantly than the level of the major chronic-stress marker cortisol, suggesting that it is a better index of acute stress. ${ }^{(34)}$

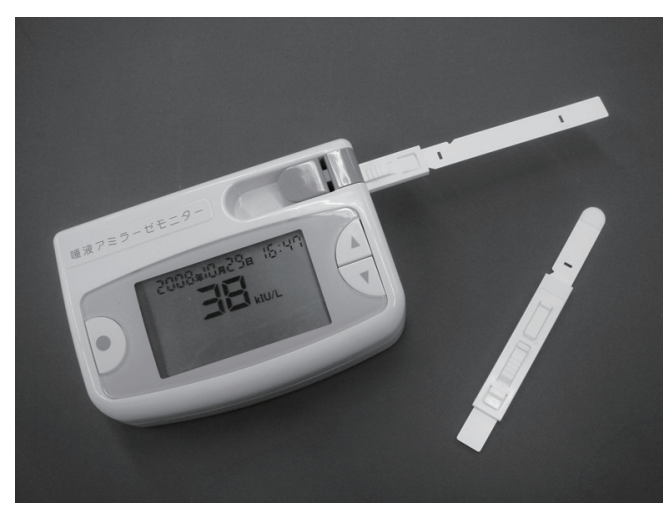

Fig. 4. Hand-held monitor for sympathetic nervous activity using salivary amylase activity (salivary amylase monitor, Nipro Co., Japan). 


\section{Conclusion}

Salivary tests have overwhelming advantages over blood tests in terms of safety and the ability for self-collection of samples. However, if we only consider these advantages alone, it is not easy to establish a diagnostic methodology for systemic diseases. It is considered that the key to the realization of a practical salivary testing is achieving greatly enhanced diagnostic accuracy for this kind of test compared with conventional technologies, or providing useful new information for medical treatment. In other words, the key is the new usage of biomarkers. The development of a rapid, low-cost and highsensitivity analytical technology for saliva samples has progressed significantly. This could promote the application of saliva testing to the rapid diagnosis of stress-induced diseases and viral infections, for which we have as yet no standard diagnosis based on an objective and quantitative index. The advent of POCT could provide a way for reaching this goal.

\section{References}

1 M. Jr. Taba, J. Kinney, A. S. Kim and W. V. Giannobile: Dent. Clin. North. Am. 49 (2005) 551.

2 D. Malamud: J. Am. Dent. Assoc. 137 (2006) 284.

3 L. A. Tabak: Ann. N. Y. Acad. Sci. 1098 (2007) 7.

4 A. Segal and D. T. Wong: Eur. J. Dent. Educ. 12 (2008) 22.

5 M. Yamaguchi and J. Arai: Measurement techniques for life sciences (Corona Publishing Co., Ltd., Tokyo, 2004) Chap. 3.

6 S. Ishikawa, S. Hashida, K. Hashinaka, K. Hirota, M. Kojima, A. Saito, A. Takamizawa, H. Shinagawa, S. Oka, K. Shimada and E. Ishikawa: J. Clin. Lab. Anal. 10 (1996) 35.

7 J. K. Champion, A. Taylor, S. Hutchinson, S. Cameron, J. McMenamin, A. Mitchell and D. Goldberg: Am. J. Epidemiol. 159 (2004) 514.

8 R. F. Vining, R. A. McGinley, J. J. Maksvytis and K. Y. Ho: Ann. Clin. Biochem. 20 (1983) 329.

9 J. M. Jr. Dabbs, R. L. Frady, T. S. Carr and N. F. Besch: Psychosom. Med. 49 (1987) 174.

10 A. Azurmendi, F. Braza, A. García, P. Braza, J. M. Muñoz, J. R. Sánchez-Martín: Horm. Behav. 50 (2006) 132.

11 M. Olff, G. J. de Vries, Y. Güzelcan, J. Assies and B. P. Gersons: Psychoneuroendocrinology 32 (2007) 619.

12 R. T. Jr. Chatterton, E. T. Mateo, N. Hou, A. W. Rademaker, S. Acharya, V. C. Jordan and M. Morrow: J. Endocrinol. 186 (2005) 77.

13 J. Hennig, U. Laschefski and C. Opper: Neuropsychobiology 29 (1994) 28.

14 C. M. Huang: Arch. Oral Biol. 49 (2004) 951.

15 S. Hu, Y. Li, J. Wang, Y. Xie, K. Tjon, L. Wolinsky, R. R. Loo, J. A. Loo and D. T. Wong: J. Dent. Res. 85 (2006) 1129.

16 B. G. Zimmermann and D.T. Wong: Oral Oncol. 44 (2008) 425.

17 B. G. Zimmermann, N. J. Park and D. T. Wong: Ann. N. Y. Acad. Sci. 1098 (2007) 184.

18 P. Groza, V. Zamfir and D. Lungu: Rev. Roum. Physiol. 8 (1971) 307.

19 M. Yamaguchi, T. Kanemori, M. Kanemaru, N. Takai, Y. Mizuno and H. Yoshida: Biosens. Bioelectron. 20 (2004) 491. 
20 R. L. Speirs, J. Herring, W. D. Cooper, C. C. Hardy and C. R. Hind: Arch. Oral Biol. 19 (1974) 747.

21 L. C. Degutis, R. Rabinovici, A. Sabbaj, R. Mascia and G. D'Onofrio: Acad. Emerg. Med. 11 (2004) 885.

22 G. K. Stookey, B. P. Katz, B. L. Olson, C. A. Drook and S. J. Cohen: J. Dent. Res. 66 (1987) 1597.

23 E. Fu, T. Chinowsky, K. Nelson, K. Johnston, T. Edwards, K. Helton, M. Grow, J. W. Miller and P. Yager: Ann. N. Y. Acad. Sci. 1098 (2007) 335.

24 S. L. Kacinko, A. J. Barnes, I. Kim, E. T. Moolchan, L. Wilson, G. A. Cooper, C. Reid, D. Baldwin, C. W. Hand and M. A. Huestis: Forensic Sci. Int. 141 (2004) 41.

25 D. C. Cullen, R. G. Brown and C. R. Lowe: Biosensors 3 (1987-1988) 211.

26 A. J. Haes and R. P. Van Duyne: J. Am. Chem. Soc. 124 (2002) 10596.

27 S. Munjal, P. Miethe, L. Netuschil, F. Struck, K. Maier and C. Bauermeister: Ann. N. Y. Acad. Sci. 1098 (2007) 486.

28 J. K. Clarke: Br. J. Exp. Pathol. 5 (1924) 141.

29 A. E. Herr, A. V. Hatch, D. J. Throckmorton, H. M. Tran, J. S. Brennan, W. V. Giannobile and A. K. Singh: Proc. Natl. Acad. Sci. USA. 104 (2007) 5268.

30 V. K. Mokkapati, R. Sam Niedbala, K. Kardos, R. J. Perez, M. Guo, H. J. Tanke and P. L. Corstjens: Ann. N. Y. Acad. Sci. 1098 (2007) 476.

31 R. F. Vining, R. A. McGinley, J. J. Maksvytis and K. Y. Ho: Ann. Clin. Biochem. 20 (1983) 329.

32 A. Stegeren, N. Rohleder, W. Everaerd and O.T. Wolf: Psychoneuroendocrinology 31 (2006) 137.

33 M. Yamaguchi, M. Deguchi, J. Wakasugi, S. Ono, N. Takai, T. Higashi and Y. Mizuno: Biosens. Bioelectron. 21 (2006) 1007.

34 N. Takai, M. Yamaguchi, T. Aragaki, K. Eto, K. Uchihashi and Y. Nishikawa: Arch. Oral Biol. 49 (2004) 963.

\section{About the Author}

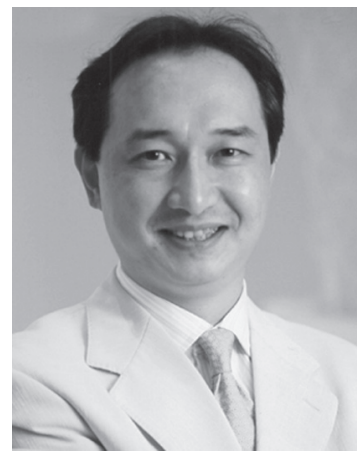

Masaki Yamaguchi was born in Nagoya, Japan, in 1963. He received the B.S. and M.S. degrees in engineering from Shinshu University, Japan, in 1985 and 1987. He joined the Research Laboratory of Brother Industries, Ltd., Japan, in 1987. He received his doctoral degree in engineering from Shinshu University in 1994 . He started as an assistant professor of Tokyo University of Agriculture and Technology, Japan in 1995. From 1999 to 2007, he was an associate professor of the Faculty of Engineering of University of Toyama, Japan. He has been working as a professor of the Graduate School of Engineering of Iwate University, Japan, since 2008.

From 1999 to 2000, he was the head researcher of Proposal-Based Immediate-Effect R\&D Promotion Program of New Energy and Industrial Technology Development Organization (NEDO) of Japan. In 2001 he received the Nikkei BP Technology Award in Medicine/Biotechnology Section by Nikkei Business Publications, Inc. for his 
achievements in the study of noninvasive measurement of blood glucose via gingival crevicular fluid. From 2002 to 2003, he worked in Linköping University in Sweden as a visiting scientist sent by the Ministry of Education, Culture, Sports, Science and Technology of Japan. He has been running a bioventure company named Bioinformation Laboratory Co. since 2004. In 2008 he was awarded the Product Prize with Nipro Corporation from the Society of Life Support Technology, Japan, for development of a noninvasive salivary amylase monitor for human stress evaluation.

He coauthored 16 books, 83 referred scientific and technical papers and 11 US patents for electromagnetic motors, medical sensors, robotics and their applications. His primary research interests focus on the development of noninvasive medical sensors and robotics. His noninvasive measurement approaches include blood glucose, human stress, skin photostress and other clinical analytical technologies. He is a member of IEEE EMBS (senior member), the Institute of Electrical Engineers of Japan (IEEJ, senior member), Japanese Society for Medical and Biological Engineering (JSMBE), and the Japan Society of Mechanical Engineers (JSME). 